\title{
Amalgamation of Clustering and Meta-heuristic Optimization Techniques for Automated MR Brain Analysis
}

\author{
Senthilkumar Natarajan, Vishnuvarthanan Govindaraj, Kannapiran Balasubramanian, \\ Pallikonda Rajasekaran Murugan, Arunprasath Thiyagarajan, Anitha Narayanan, Deny John \\ Samuvel,
}

\begin{abstract}
Interest in computer-assisted image analysis in increasing among the radiologist as it provides them the additional information to take decision and also for better disease diagnosis. Traditionally, MR image is manually examined by medical practitioner through naked eye for the detection and diagnosis of tumor location, size, and intensity; these are difficult and not sufficient for accurate analysis and treatment. For this purpose, there is need for additional automated analysis system for accurate detection of normal and abnormal tumor region. This paper introduces the new semi-automated image processing method to identify the brain tumor region in Magnetic Resonance Image (MRI) using $c$ means clustering technique along with meta-heuristic optimization, based on Jaya optimization algorithm. The resultant performance of the proposed algorithm $(F C M+J A)$ is examined with the help of key analyzing parameters, MSE-Mean Square Error, PSNR-Peak Signal to Noise Ratio, DOI-Dice Overlap Index and CPU memory utilization. The experimental results of this method show better and enhanced tumor region display in reduced computation time.
\end{abstract}

Keywords: Jaya Algorithm (JA), Tumor detection, Fuzzy C Means Clustering, Meta-heuristic Optimisation.

\section{INTRODUCTION}

$\mathrm{W}$ orldwide, several researches reveal that death rate of children as well as in adults increase due to brain tumor and this has become a great concern of the time. So it turned to be a need for the hour to have automated detection system for

Revised Manuscript Received on December 15, 2019.

* Correspondence Author

Senthilkumar Natarajan*, Department of Electronics and Communication Engineering, Kalasalingam Academy of Research and Education, Virudhunagar, India. Email: senthil.analyst86@gmail.com

Vishnuvarthanan Govindaraj, Department of Biomedical Engineering, Kalasalingam Academy of Research and Education Virudhunagar, India. Email: gvvarthanan@gmail.com

Kannapiran Balasubramanian, Department of Electronics and Communication Engineering, Dr.Mahalingam College of Engineering and Technology Pollachi, India. Email: kannapiran79@gmail.com

Pallikonda Rajasekaran Murugan, Department of Electronics and Communication Engineering, Kalasalingam Academy of Research and Education, Virudhunagar, India. Email: m.p.raja@klu.ac.in

Arunprasath Thiyagarajan, Department of Biomedical Engineering, Kalasalingam Academy of Research and Education Virudhunagar, India. Email: arun.aklu@gmail.com

Anitha Narayanan, Department of Electronics and Communication Engineering, Kalasalingam Academy of Research and Education, Virudhunagar, India. Email: anithaa06@gmail.com

Deny John Samuvel, Department of Electronics and Communication Engineering, Kalasalingam Academy of Research and Education, Virudhunagar, India. Email: prof.j.deny@gmail.com

better interpretation of diseases by the physicians at the early stage. Generally, a mass of abnormal lump or affected cells that grows and spreads uncontrollably in and around the brain region is called brain tumor. Magnetic Resonance imaging technique has received notable attention among the medical practitioner because of its high-resolution imaging feature. MRI is widely used for the analysis of human parts brains, spinal cord, bones and Joints etc... Difficulty in detection of small size tumors, similarity between the affected and unaffected regions, and limited time for the computation of large slice of images urges the radiologists to use various soft computation techniques for analyzing the MRI. Globally, numerous soft computation approaches are introduced by researchers for brain tumor detection using image analyzing techniques. In Section II, A detailed literature study on C-Means algorithm and novel Jaya Algorithm is given. SECTION III elaborates the algorithm proposed. Obtained results are stated briefly using parameters in SECTION IV. Results and future avenues were discussed in SECTION V.

\section{LITERATURE REVIEW}

In the literature, there are substantial numbers of research work carried with C-Means clustering due to its proved efficiency and less computational complexity. In case of Jaya Algorithm as it was developed recently in 2015, there is no much exploration, particularity in the field of brain pathology diagnosis. For the image segmentation, Yunjie Chein et al. [1] discussed about spatially constrained hierarchy Fuzzy C-Means (FCM) method. The hierarchy strategy used in the method increases the complexity of process. RobustLearning FCM clustering algorithm was proposed by Miin-Shen Yang and Yessica Nataliani [2], in which the computational time is high due to more number of iterations. An automated segmentation of tumor region using optimization and clustering techniques for three (T1, T2 \& Flair) image modality was developed by Vishnuvarthanan Govindaraj et al. [3], requires improvement in segmentation accuracy. Deepak Ranjan et al. [4] formulated the pathological brain detection systems (PBDSs) using Jaya Algorithm and extreme learning machine combination for the identification of tumor region, and this method works well on 3 benchmark data sets. Suresh Chandra Satapathy et al. [5] worked on a methodology that encapsulates Jaya algorithm and Otsu's at pre-processing and post-processing stage of

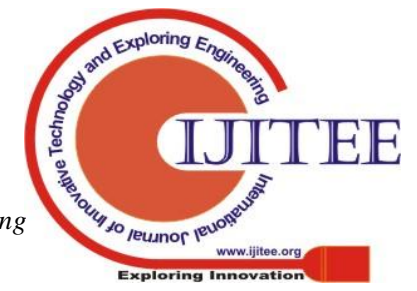


image analysis. Later, Kanwarpreet Kaur et al. [6] have developed Graphical User Interface (GUI) using Neural Network Ensemble and Jaya algorithm for segmentation and also for extraction of pathology region. A GUI approach was proposed for acute classification of leukemia by Jothi et al. [7], which works only on single model data set. Smart pathological brain detection using Extreme Learning Machine and Jaya Algorithms proposed by Vishnuvarthanan et al. [8] requires improvement of time complexity. Shuihua Wang et al. [9] proposed abnormal breast classification method in mammogram images using forward feed network and Jaya Algorithm, and it is applied to train the classifier network, which requires improvement in accuracy. Rao has developed an algorithm for the optimization without the requirement of algorithm-described parameters to solve constrained and unconstrained problems. [10]. This paper addresses the above said limitations by combining the simple and novel algorithms, Fuzzy C-Means clustering (FCM) and Jaya algorithm.

\section{METHODOLOGY}

First, Image is enhanced and noise removing filters are applied at pre-processing stage. Next, tumor region is clustered by FCM technique, then the Jaya algorithm is used to optimize the clustered region. At last, results were analyzed with benchmark data. The proposed workflow consists of 4 modules and each is elaborated below.

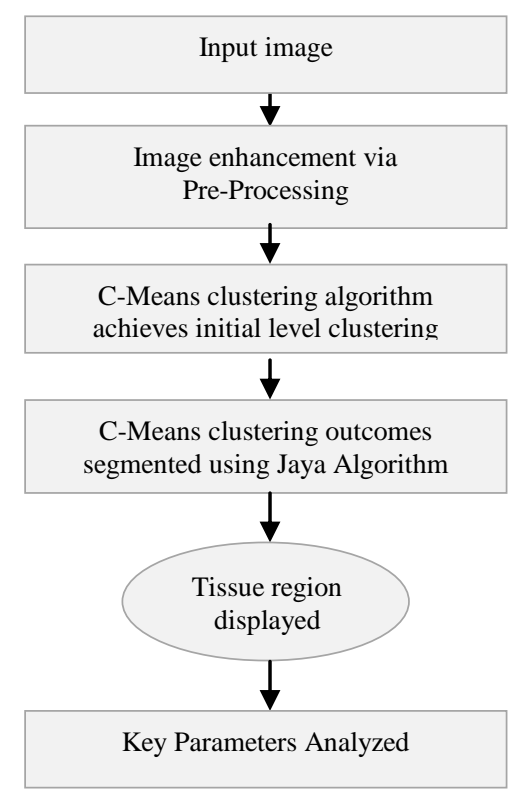

Fig. 1. Outline of workflow

\section{A. Pre-Processing}

In medical image analyzing procedure, pre-processing is the initial and most important task as it enhances the corrupted image's sharpness and it also reduces the noise content to produce high-quality image. In this stage, we resize the image's Region of Interest (ROI) by 256 x 256 and apply Gaussian filter to smoothing, remove noises and also to preserve the edges without any loss in information.

\section{B. Fuzzy C-Means clustering}

FCM is deployed in this paper in the clustering stage owing to its flexibility and its ability to augment more information in the input images while processing compared to other clustering algorithms. FCM uses fuzzy logic and fuzzy set theory to produce optimal number of clustering. Here, each data set (or pixels) is grouped into $\mathrm{n}$ clusters with assigned membership degree.

Algorithm for Fuzzy c means clustering:

- Step 1: Initialize the cluster centre point randomly and set iteration $\mathrm{i}=0$.

- Step 2: Assign membership weightage to all the data points in a cluster.

- Step 3: Increase the iteration count by 1 .

- Step 4: Update the cluster centre and membership weightage of each data points present in the clusters.

- Step 5: If the cluster centre does not change with the previous value, then end the process or repeat the step 3 until desired centre is fixed.

On each iteration, the cluster centre points move towards the correct centroid data points by minimizing the below objective function.

$$
F_{f_{c m}}(U, M)=\sum_{i=1}^{c} \sum_{j=1}^{N} U_{i j}^{m}\left\|{w_{i}}_{i} c_{i}\right\|^{2}
$$

Where,

Data points for $\mathrm{N}$ pixels is

$$
X=\left\{\boldsymbol{x}_{s}\right\}_{1}^{N}
$$

Membership weightage is

$$
W=w_{i j} \in[0,1]
$$

$\mathrm{W}_{\mathrm{ij}}$ represents the weight component of $\mathrm{i}^{\mathrm{th}}$ data point in the $\mathrm{j}^{\text {th }}$ cluster with the value between $\{0,1\}$.

The centroid of the each cluster is updated by reducing the distance between the cluster centres $\mathrm{C}_{\mathrm{i}}$ and the $\mathrm{i}^{\text {th }}$ pixel $\mathrm{x}_{\mathrm{s}}$

$$
\boldsymbol{C}_{i}=\frac{\sum_{s=1}^{N} \boldsymbol{U}_{i j}^{m} \boldsymbol{X}_{s}}{\sum_{s=1}^{N} \boldsymbol{U}_{i j}^{m}}
$$

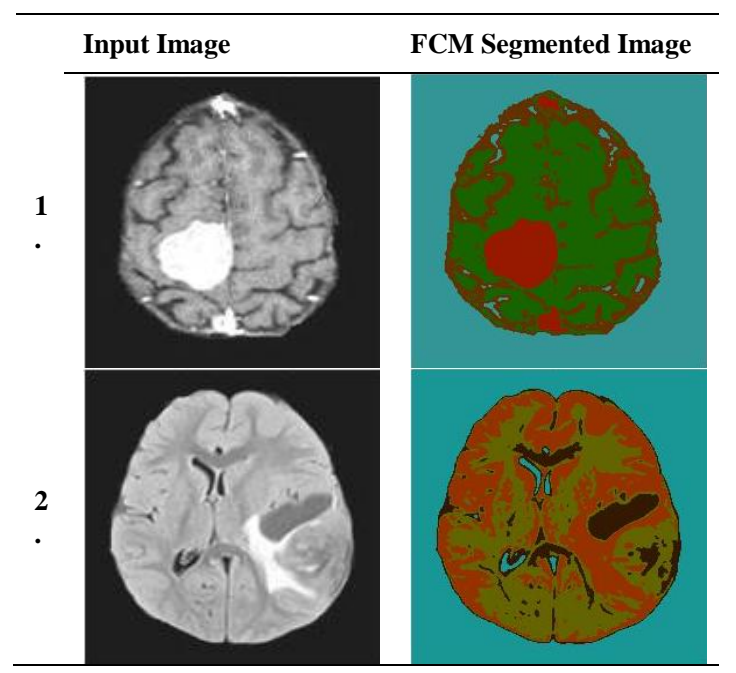

Fig. 2. Output image for FUM clustering

\section{Published By:}

Blue Eyes Intelligence Engineering 
In literature, it is proved that the FCM based tumor region detection methods are more efficient and highly reliable compared to the K-Means clustering. On considering these, we have applied the FCM for our clustering process.

\section{JAYA Algorithm-Optimization}

FCM algorithm takes number of iteration to reduce the clusters membership degree and also to finalise the exact cluster points, and this may reduce the overall efficiency. To improve the over-all performance, we combined FCM with the Meta-heuristic optimization techniques.

A novel optimization technique Jaya optimization algorithm is employed to optimize the clustered image considering its effective and speedy computation features. This algorithm is successfully applied to the various constrained and unconstrained problems across domains universally and received wide acceptance among the researchers. In 2015, Jaya Algorithm was introduced by Venkata Rao [7]. This algorithm is derived from his previous optimization named as TLBO-Teaching and Learning Based Optimization, which also optimizes the objective function without any predefined parameters required by the underlying algorithm.

On Comparing with TLBO method, Jaya algorithm seems to be very simple and fast in execution as it does not need two phases namely teaching and learning phase for the computation. The advantage of Jaya Algorithm is Algorithm-Specific Parameters (ASP) are not required, and they are only in the need of general parameters like maximum iterations required to proceed, total population size and objective function to solve. The specialty of this algorithm is that on every end of iteration the desired variable is updated and it moves closer to the favorable solution, and eliminates or moving away from the unfavorable solution.

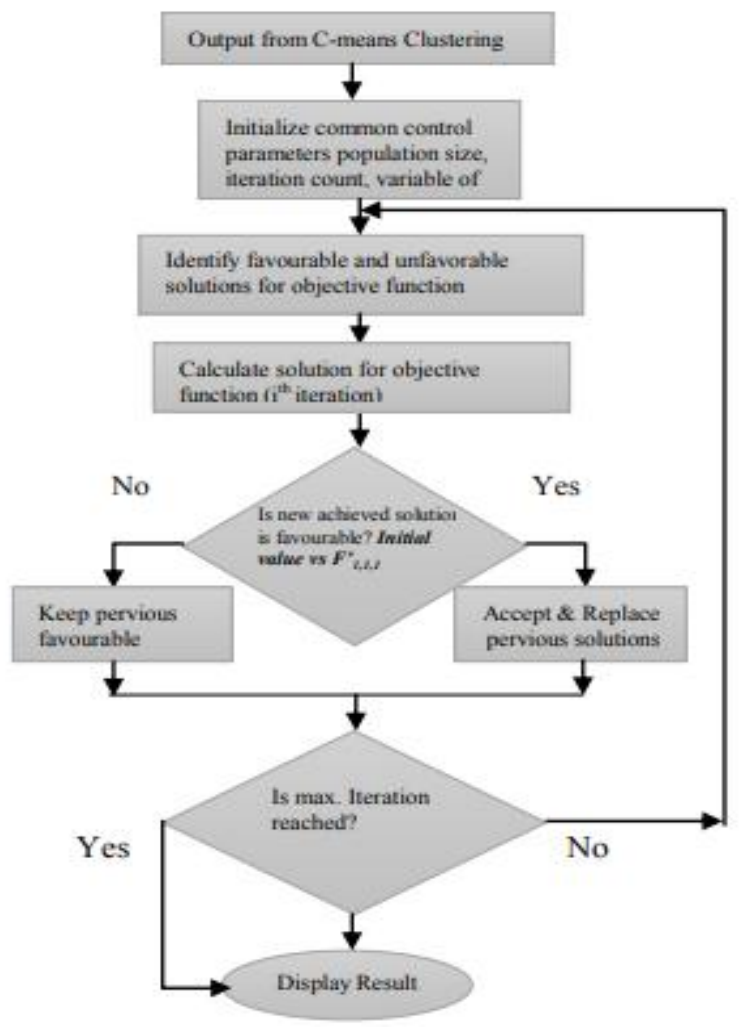

Fig. 3. Flow chart -JAYA algorithm
We assume that $p(x)$ is the objective function desirable for processing. At any iteration $i$, if there are ' $c$ ' variables in number (i.e., blocks based on clustering, where $n=1,2, . . c$ ), and ' $n$ ' specifies the candidates count (i.e. size of the population $\left.p=0,1,2, \ldots, S{ }_{(255)}\right)$. Both the favorable and unfavorable candidate help to determine the objective function $p(x)$ among the total population, and they are indicated as $p(x)_{\text {fav }}$ and $p(x)_{\text {unfav }}$.

At the instance of first step, the value $P_{c, s, i}$ represents the $\mathrm{c}^{\text {th }}$ variable denoting the $s^{\text {th }}$ candidate at the time of $i^{\text {th }}$ iteration, and as per the following Eq.(5). The parameters rd (i,c,1) and rd (i,c,2) are the independently developed for each design variable at each iteration cycle. The value of the parameters is in $[0,1]$.

$P_{c, s, i}^{\prime}=P_{c, s, i}+r d_{1, c, i}\left(P_{c, f a v, i}-\left|P_{c, s, i}\right|\right)-r d_{2, c, i}\left(P_{c, u n f a v, i}-\left|P_{c, s, i}\right|\right)$

From the above equation, the first term $r d_{l, c, i}\left(P_{c, f a v, I}\right.$ - $\left.\left|P_{c, s, i}\right|\right)$ shows the ability of desired objective moves towards favourable condition (denoted with + symbol) and the second term $r d_{2, c, i}\left(P_{c, \text { unfav }, i^{-}}\left|P_{c, s, i}\right|\right)$ illustrates that the objective function is moving away from the unfavorable condition (denoted with - symbol). On next iteration, the new favorable and unfavorable values are replaced (given as input), and the iteration continued till it reaches either its final count or it reaches favourable solution for the desired objective function as shown in Eq (6).

$$
P_{c, s, i}^{\prime \prime}=P_{c, s, i}+r d_{1, c, i}\left(P_{c, f a v, i}^{\prime}-\left|P_{c, s, i}\right|\right)-r d_{2, c, i}\left(P_{c, f u n a v, i}^{\prime}-\left|P_{c, s, i}\right|\right)
$$

The proposed Jaya algorithm applied on the outcome of FCM clustering process, where it takes only few iterations to minimize the desired objective function. The robustness of segmentation improved significantly and makes the method more accurate in terms of segmented output. 


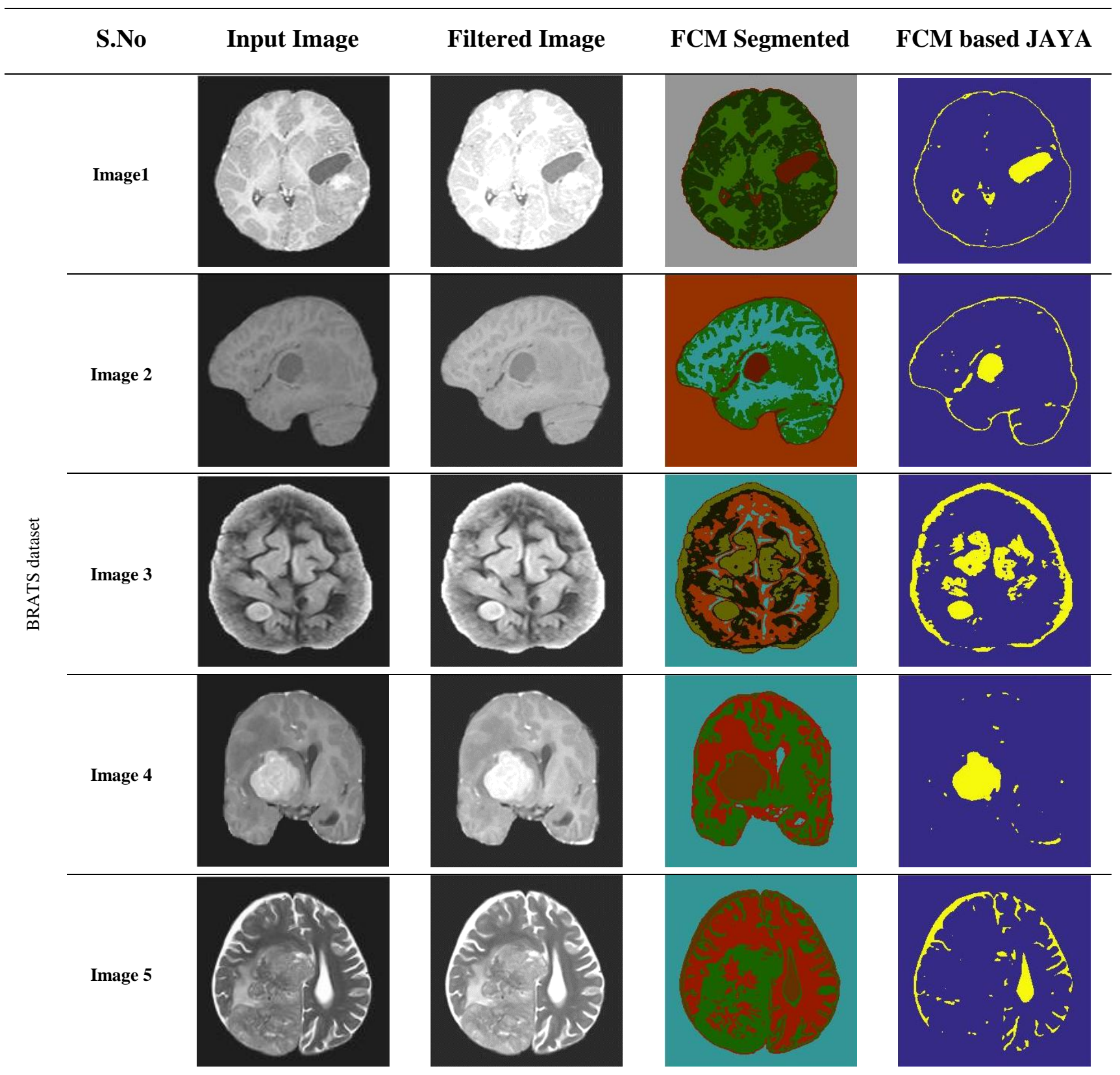

Fig. 4. Proposed FCM-JA method outcome.

\section{RESULT AND DISCUSSION}

The proposed C-means clustering based Jaya Optimization method has been executed with MATLAB. Furthermore, the proposed method overcomes the drawbacks of computation time and accuracy by implementing novel optimization techniques, JA along with FCM. The segmented results were evaluated by key parameters, MSE-Mean Squared Error. PSNR-Peak Signal to Noise ratio, DOI, computation time, and system memory requirement. The proposed algorithm performs better endue on data processing time with low system memory utilization compared to the benchmark segmentation methods. Table 1, sums up the key evaluation parameters for five images belonging to clinical data set.

Table I: Key metrics analysis of the FCM - Jaya algorithm

\begin{tabular}{|c|c|c|c|c|c|}
\hline Particulars & Image 1 & Image 2 & Image 3 & Image 4 & Image 5 \\
\hline MSE & 2.0102 & 1.2701 & 1.0791 & 1.0060 & 1.0618 \\
\hline PSNR (Db) & 5.0983 & 8.9538 & 7.8000 & 8.1048 & 7.8701 \\
\hline TC (\%) & 2.4865 & 2.6807 & 2.228 & 3.7855 & 2.3805 \\
\hline DOI (\%) & 3.9827 & 4.2281 & 3.6453 & 5.4920 & 3.8456 \\
\hline $\begin{array}{c}\text { Computational } \\
\text { Time (in seconds) }\end{array}$ & 84.41 & 56.82 & 67.87 & 68.73 & 56.42 \\
\hline $\begin{array}{c}\text { Memory } \\
\text { requirement (in } \\
\text { bytes) }\end{array}$ & $2.21 \mathrm{e}+09$ & $2.20 \mathrm{e}+09$ & $2.27 \mathrm{e}+09$ & $2.19 \mathrm{e}+09$ & $2.21 \mathrm{e}+09$ \\
\hline
\end{tabular}

Published By:






\section{CONCLUSION}

Universally, many researchers developing many optimization methods to detect the tumor region automatically from large slice of images consider the huge scope for efficient optimization techniques, in acquiescence to this, the paper propounds a novel combination of JAYA algorithm. We reduced the computation time, complexity and improved the performance of the clustering outcome by combining FCM with JA. The combined performance of the (FCM + JAYA) algorithm shows endue results compared to the segmentation results obtained by individual FCM method and is evident from the obtained superior numbers of the analyzing criteria mentioned in Table I. In future, we will extend the study a) with large and multimodal datasets b) and to enhance the performance of the segmentation process.

\section{ACKNOWLEDGMENT}

The authors gratefully acknowledges the staffs of department of Electronics and Communication Engineering of Kalasalingam University, Krishnankovil, India for their valuable time and support for accomplishing this research a success. The authors also thank Dr.K.G.Srinivasan, MD, RD, Consultant Radiologist and Dr.K.P.UshaNandhini, DNB, KGS Advanced MR \& CT Scan - Madurai, Tamilnadu, India, for supporting the research with the patient information.

\section{REFERENCES}

[1] YangMiin-Shen, "Robust-learning fuzzy c-means clustering algorithm with unknown number of clusters," Pattern Recognition, vol. 71, pp. 45-59, 2017.

[2] Yunjie Chen, Jian Li, Hui Zhang, Yuhui Zheng, Byeungwoo Jeon, and Qingming Jonathan $\mathrm{Wu}$, "Non-local-based spatially constrained hierarchical fuzzy C-means method for brain magnetic resonance imaging segmentation IET Image Process," The Institution of Engineering and Technology, vol. 10, pp. 865-876, 2016.

[3] Vishnuvarthanan Govindaraj,Pallikonda Rajasekaran, Murugan, "A complete automated algorithm for segmentation of tissues and identification of tumor region in T1, T2, and FLAIR brain images using optimization and clustering techniques," Wiley Periodicals, vol. 24 , pp. 313-325, 2014.

[4] Deepak Ranjan Nayak, Ratnakar Dash, and Banshidhar Majhi, "Development of pathological brain detection system using Jaya optimized improved extreme learning machine and orthogonal ripplet-II transform," Springer Science+Business Media, 2017.

[5] Suresh Chandra Satapathy, and Venkatesan Rajinikanth, "Jaya Algorithm Guided Procedure to Segment Tumor from Brain MRI," Hindawi Journal of Optimization, Article ID 3738049, 12 pages, 2018.

[6] Kanwarpreet Kaur, Gurjot Kaur Walia, and Jaspreet Kaur, "Neural Network Ensemble and Jaya Algorithm Based Diagnosis of Brain Tumor Using MRI Images," Journal of the Institution of engineers(India): Series B, vol 99. PP 509-517, 2018.

[7] Yu-Dong Zhang, Guihu Zhao, Junding Sun, Xiaosheng Wu, Zhi-Heng Wang, Hong-Min Liu, Vishnu Varthanan Govindaraj, Tianmin Zhan, \& Jianwu Li, "Smart pathological brain detection by synthetic minority oversampling technique, extreme learning machine, and Jaya algorithm," Springer Science+Business Media, LLC 2017.

[8] Shuihua Wang, Peng Chen, Aijun Liu, Ravipudi Venkata Rao, Yudong Zhang, \& Ling Wei, "Abnormal Breast Detection in Mammogram Images by Feed-forward Neural Network Trained by Jaya Algorithm," Fundamenta Informaticae, vol. 151, pp.191-211, 2017.

[9] R Venkata Rao, "Jaya: A simple and new optimization algorithm for solving constrained and unconstrained optimization problems,", International Journal of Industrial Engineering Computations, vol 7, PP.19-34, 2016.

[10] R.V. Rao, V.J. Savsani, \& D.P Vakharia, "Teaching-learning-based optimization: A novel method for constrained mechanical design optimization problems," Computer-Aided Design, vol 42 issue3, PP 303-315, 2011.

[11] G. Vishnuvarthanan, M.P. Rajasekaran, P. Subbaraj, and A. Vishnuvarthanan, "An unsupervised learning method with a clustering approach for tumor identification and tissue segmentation in magnetic resonance brain images," Applied Soft Computing, vol. 38, pp 190-212, 2016. DOI: https://doi.org/10.1016/j.asoc.2015.09.016.

[12] Jeetashree Aparajeeta, Pradipta Kumar Nanda, Niva Das, "Modified possibilistic fuzzy C-means algorithms for segmentation of magnetic resonance image," Applied Soft Computing, vol.41 pp. 104-119, 2016.

[13] J. C. Dunn, "A fuzzy relative of the ISODATA process and its use in detecting compact well separated clusters," Journal of Cybernetics, vol. 3 , no. 3, pp. 32-57, 1974

[14] J. C. Bezdek, "A convergence theorem for the fuzzy ISODATA clustering algorithms," IEEE Transaction on Pattern Analysis and Machine Intelligence, vol. 2, no. 1, pp. 1-8, 1980.

[15] Ming-Chuan Hung and Don-Lin Yang, "An Efficient Fuzzy C-Means Clustering Algorithm IEEE International Conference on Data Mining. doi:10.1109/icdm.2001.989523

\section{AUTHORS PROFILE}

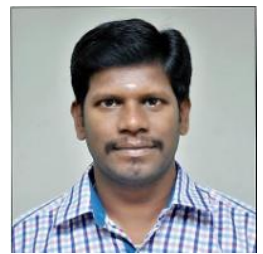

Mr. N. Senthilkumar is a research scholar in Kalasalingam Academy of Research and Education. $\mathrm{He}$ is currently working on optimization techniques for medical image processing.

He completed under graduation (B.E) in Electronics and Communication Engineering from V.R.S College of Engineering and Technology, affiliated to Anna University, Villupuram, India and also pursued Master degree in Digital Communication and Networking from Bharath Institute of Higher Education and Research, Chennai. His areas of interests are image and signal processing, optimization and soft computing techniques.

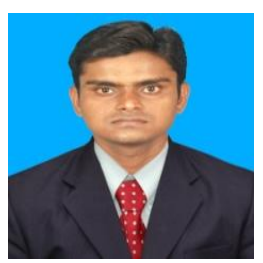

Dr. G .Vishnuvarthanan, born in 1986, has research stints in the avenues of medical image processing and artificial intelligence. He was awarded $\mathrm{PhD}$ in 2015 and bachelor's degree in Instrumentation and Control Engineering by 2007, and Master's Degree in VLSI by 2009. Currently working in Kalasalingam Academy of Research and Education as Associate Professor in Biomedical Engineering Department. He is highly knowledge in teaching and research with more than a decade of experience in teaching and research. He is member of ISTE and has 17 SCI Journal and 20 International Publications which have been indexed in SCOPUS database.

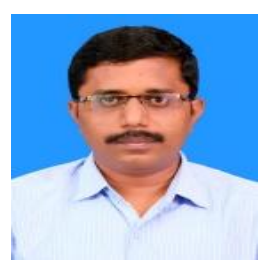

Dr. B. Kannapiran completed his B.E Degree in Instrumentation and Control Engineering in 2001 and also received M.E degree in Applied Electronics from Madurai Kamaraj University. He received the Ph.D Degree in Information and Communication Engineering from Anna University, Chennai in 2013. He is currently working as a Professor a Dr.Mahalingam College of Engineering and Technology, Pollachi, Tamilnadu, India. He guided more than 7 Ph.D scholars out of which 4 Ph.D scholars have completed their research work. Soft computing, fault diagnosis, Intrusion detection in wireless networks and sensor designs are his area of research interest. 


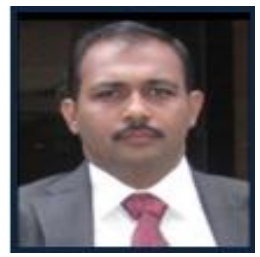

Dr. Pallikonda Rajasekaran Murugan, is working as professor in Kalasalingam Academy of Research and Education since 2012. He graduated in Electronics and Instrumentation in 2001 from Shanmuga college of Engineering, Thanjavur and received his M.Tech at SASTRA University in 2002. He received the $\mathrm{Ph}$.D. Degree from, Anna University, Chennai, India. He has rich experience in the field of bio image and signal processing. Under his guidance more than 8 scholars completed their doctorates across various fields like Image Processing, Biomedical Instrumentation, and Wireless Sensor Network. He has strong track record of publications with more than 50 papers published in various journals and conferences. He holds membership in various technical bodies like ISTE, IEEE, IE, APCBEES, IAENG and IACSIT.

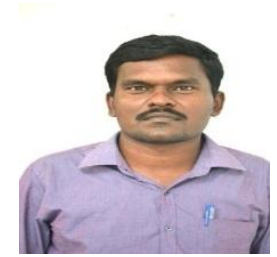

Dr. T. Arunprasath receive the Ph.D in Electronics and Communication Engineering from Kalasalingam University in 2015. He completed his Master Degree in Applied Electronics in Mohamed Sathak Engineering College in 2009 and Bachelor Degree in Syed Ammal Engineering College in the year 2006. He is presently working as Associate Professor in Department Biomedical Engineering at Kalasalingam Academy of Research and Education. He is keen in the research areas like image processing biomedical, image segmentation techniques and cloud computing. Dr.T.Arunprasath published 16 technical journals and 20 technical papers in refereed conferences in his research areas and he is fellow member of ISTE.

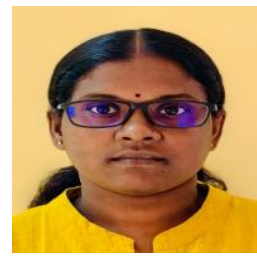

Dr. Anitha. $\mathbf{N}$ awarded her PhD degree from Kalasalingam Academy of Research and Education (Kalasalingam University) in 2019 for her significant contribution in the area of medical image processing. She completed M.E in Digital Communication and Networking in 2015 and B.E in Electronics and Communication Engineering in 2006. She has three years of work experience as software programmer and two years in teaching, also an expertise in students' counselling. She is an active IEEE Member of Pothigai Subsection and IEEE Solid-State Circuits Society Member of IEEE Madras section from March 2018.



Dr. J. Deny is an Associate Professor in the Department of ECE at Kalasalingam Academy of Research and Education. He received his Ph.D. degree from Bharath Institute of Higher Education and Research in the year 2017. He did his M.Tech Digital Communication and Networks at Kalasalingam Academy of Research and Education in 2012. In the year 2010, He completed his graduation in Electronics and Communication Engineering and in 2012, he received M.Tech degree in Digital Communication and Networking. He plays a key role as the editorial and reviewer board for 30 reputed journal and also holds membership in technical bodies namely, IAAM, CSTA, SDIWC, IAEME, IAENG, ACM and IACSIT. He has deep involvement in the research areas like Network Security and image processing. 